\title{
Regime shifts in a social-ecological system
}

Steven J. Lade, Alessandro Tavoni, Simon A. Levin, Maja Schlüter

February 2013

Centre for Climate Change Economics and Policy Working Paper No. 125

Grantham Research Institute on Climate Change and the Environment Working Paper No. 105 
The Centre for Climate Change Economics and Policy (CCCEP) was established by the University of Leeds and the London School of Economics and Political Science in 2008 to advance public and private action on climate change through innovative, rigorous research. The Centre is funded by the UK Economic and Social Research Council and has five inter-linked research programmes:

1. Developing climate science and economics

2. Climate change governance for a new global deal

3. Adaptation to climate change and human development

4. Governments, markets and climate change mitigation

5. The Munich Re Programme - Evaluating the economics of climate risks and opportunities in the insurance sector

More information about the Centre for Climate Change Economics and Policy can be found at: http://www.cccep.ac.uk.

The Grantham Research Institute on Climate Change and the Environment was established by the London School of Economics and Political Science in 2008 to bring together international expertise on economics, finance, geography, the environment, international development and political economy to create a worldleading centre for policy-relevant research and training in climate change and the environment. The Institute is funded by the Grantham Foundation for the Protection of the Environment and the Global Green Growth Institute, and has five research programmes:

1. Global response strategies

2. Green growth

3. Practical aspects of climate policy

4. Adaptation and development

5. Resource security

More information about the Grantham Research Institute on Climate Change and the Environment can be found at: http://www.lse.ac.uk/grantham.

This working paper is intended to stimulate discussion within the research community and among users of research, and its content may have been submitted for publication in academic journals. It has been reviewed by at least one internal referee before publication. The views expressed in this paper represent those of the author(s) and do not necessarily represent those of the host institutions or funders. 


\title{
Regime shifts in a social-ecological system
}

\author{
Steven J. Lade • Alessandro Tavoni - Simon A. Levin • Maja Schlüter
}

Received: date / Accepted: date

\begin{abstract}
Ecological regime shifts are rarely purely ecological. Not only is the regime shift frequently triggered by human activity, but the responses of relevant actors to ecological dynamics are often crucial to the development and even existence of the regime shift. Here, we show that the dynamics of human behaviour in response to ecological changes can be crucial in determining the overall dynamics of the system. We find a social-ecological regime shift in a model of harvesters of a common-pool resource who avoid over-exploitation of the resource by social ostracism of non-complying harvesters. The regime shift, which can be triggered by several different drivers individually or also in combination, consists of a breakdown of the social norm, sudden collapse of co-operation and an over-exploitation of the resource. We use the approach of generalised modelling to study the robustness of the regime shift to uncertainty over the specific forms of model components such as the ostracism norm and the resource dynamics. Importantly, the regime shift in our model does not occur if the dynamics of harvester behaviour are not included in the model. Finally, we sketch some possible early
\end{abstract}

Steven J. Lade · Maja Schlüter

Stockholm Resilience Centre, Stockholm University,

Kräftriket 2B, 11419 Stockholm, Sweden

E-mail: steven.lade@stockholmresilience.su.se

Steven J. Lade

NORDITA, KTH Royal Institute of Technology and

Stockholm University, Roslagstullsbacken 23,

10691 Stockholm, Sweden

Alessandro Tavoni

Grantham Research Institute, London School of Economics, London WC2A 2AZ, United Kingdom

Simon A. Levin

Department of Ecology and Evolutionary Biology, Princeton University, Princeton, NJ 08544, USA warning signals for the social-ecological regime shifts we observe in the models.

Keywords Regime shifts - tipping points - early warning signals · bifurcation - generalised modelling · social-ecological system

\section{Introduction}

Many ecological systems can undergo large, sudden and long-lasting changes in structure and function (Scheffer et al 2001). Such changes, often called regime shifts ${ }^{1}$ or critical transitions (Scheffer et al 2009), have been found in a range of ecological systems, including eutrophication of freshwater lakes, soil salinisation, degradation of coral reefs, collapse of fisheries and encroachment of bushland (Biggs et al 2012a).

Most ecological systems, and especially those systems that are at risk of sudden nonlinear changes such as regime shifts, are subject to influence by humans (Millennium Ecosystem Assessment 2005). Furthermore, humans not only influence the ecological system but also adapt their behaviour in response to ecological changes (Folke et al 2010). However, many traditional ecological modelling approaches reduce the social subsystem to a simple driver, such as fishing pressure or resource extraction rate (Schlüter et al 2012a). Mirroring this problem, in bioeconomic models of the optimal management of renewable resources the descriptions of resource dynamics are often very simple, with neither

1 In this article, we use the term 'regime shift' in a social as well as an ecological context. We intend the term 'regime shift' to be understood in such a social context as not (necessarily) a political regime change but rather any recognisably sudden, large and persistent change in the behaviour of relevant actors. 
resource nor management strategy capable of regime shifts (with exceptions including recent work from ecological economics such as Crépin and Lindahl 2009; Horan et al 2011).

Here, we show that modelling changes in the behaviour of the humans who interact with the ecological system is crucial to understanding regime shifts in the ecological populations. In particular, a central result is that such systems, often referred to as socialecological systems (Carpenter et al 2009), can display regime shifts that are absent from the ecological subsystem in isolation. We find that even a non-linear linkage between completely linear social and ecological subsystems, which have no regime shifts of their own, can induce regime shifts in the coupled system.

To develop these results, we analyse a social-ecological system that captures essential properties of a class of systems encountered frequently in natural resource management: a common-pool resource, for which harvesters choose between a community-efficient co-operation or a self-interested defection that leads to over-harvesting, together with a social mechanism that encourages but does not guarantee co-operation (Ostrom 1990, 2006). We represent this system using two types of models: a generalised model, where the details of the processes in the system are left unspecified; and simulation models based on the model of Tavoni et al (2012) (hereafter referred to as the TSL model) that have functional forms fully specified, as is necessary to perform time series simulations.

We search for possible regime shifts in this socialecological system by analysing the fold bifurcations of the models. Fold bifurcations, one of a family of precisely mathematically defined qualitative changes in the dynamical behaviour of a system (Kuznetsov 2010), can lead to regime shifts (Scheffer et al 2001). Bifurcation analysis of the generalised model therefore permits general statements about the presence and robustness of regime shifts over a wide range of systems. This generality is useful when analysing social-ecological systems, in which the specific functional forms required by simulation models can be difficult to determine. Bifurcation analysis of simulation models allows us to test the generalised results in specific cases and to predict the presence and effects of regime shifts with respect to specific parameters of the simulation model.

Since regime shifts are sudden, persistent and often have significant consequences, early warning signals for an impending regime shift would be highly desirable. Early warning signals have recently been developed for regime shifts in ecological and physical systems (Scheffer et al 2009, 2012). We perform preliminary investigations on the possibility of using these early warn- ings for regime shifts in social-ecological systems. We use the conventional variance and autocorrelation indicators (Dakos et al 2012b) as well as the generalised modelling-based early warning signal (Lade and Gross 2012).

Section 2 describes in greater detail the models and the methods used to analyse them, including bifurcation diagrams and generalised modelling for both bifurcation analysis and early warning signals as well as conventional early warning signals. Section 3 presents the results of these bifurcation and early warning signal analyses, and in Section 4 we discuss their implications for modelling studies and for governance of socialecological systems. Concluding remarks are presented in Section 5.

\section{Methods}

We begin by introducing background theory and the analytical tools we propose to use in our analysis, then describe the model to which they will be applied.

\subsection{Bifurcation theory}

Bifurcation theory describes sudden, qualitative changes in the dynamical behaviour of systems. Specifically, a bifurcation of a dynamical system occurs when a small, smooth change in a parameter of the dynamical system causes a qualitative change to the dynamics of the system (Kuznetsov 2010). In this article, we analyse the bifurcations of our social-ecological models to understand qualitative changes in their behaviour.

Regime shifts are a type of qualitative change in behaviour that can be caused by bifurcations (Kuehn 2011). One important type of regime shift-inducing bifurcation is the fold bifurcation (Scheffer et al 2001). In a fold bifurcation, a stable fixed point collides with an unstable fixed point and both fixed points disappear (Fig. 1). A system initially on or near the stable fixed point will undergo a sudden and possibly large change in state to a distant attractor (in Fig. 1 this is another stable fixed point). This change is also persistent, as the parameter must often be changed to a significantly different value in order for the system to transition back to the first fixed point. Mathematically, fold bifurcations occur (in the ordinary differential equations we use here) when a real eigenvalue of the Jacobian matrix at the fixed point passes through zero. The Jacobian matrix is a matrix of derivatives that characterises the dynamics of the system in the vicinity of the fixed point. 


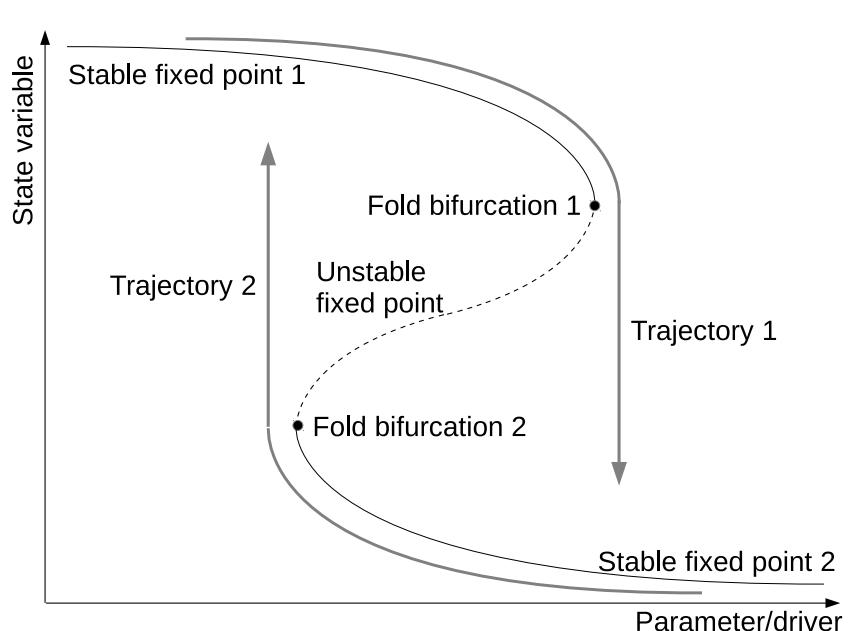

Fig. 1 Bifurcations and regime shifts. Fold bifurcations occur when a stable (solid lines) and an unstable (dashed line) fixed point collide, and lead to regime shifts. In the pair of fold bifurcations sketched here (manually, without computer simulation), a system on stable fixed point 1 will, upon the driver passing fold bifurcation 1, undergo a sudden and large shift to stable fixed point 2 (trajectory 1 ). This change is persistent, because the parameter must be returned to a value below fold bifurcation 2 (if this is possible) in order for the system to return to its original state, in another regime shift (trajectory 2).

Another important type of bifurcation that can also be detected with a generalised modelling analysis is the Hopf bifurcation, which often (in the case of supercritical Hopf bifurcations) lead to a transition to oscillatory dynamics. A Hopf bifurcation occurs when the Jacobian matrix at a fixed point has a complex conjugate pair of eigenvalues that pass through the imaginary axis (that is, have zero real part) (Kuznetsov 2010).

For simulation models of dynamical systems, where the forms of all interactions and the values of all parameters are known, numerical continuation software are often used to locate and track bifurcations. Here, we used the software XPPAUT (Ermentrout 2011) to plot bifurcation diagrams of the form of Fig. 1, where the values of a parameter or 'driver' are plotted on the horizontal axis, and the stable and unstable fixed points of the system corresponding to each value of the driver on the vertical axis.

\subsection{Generalised modelling for bifurcation analysis}

Bifurcation diagrams give great insight into possible qualitative behaviours of the system. Frequently, however, the forms of interactions and the values of parameters in the model on which the bifurcation analysis was performed cannot be accurately known. In a related problem, if functional forms are chosen in a model, there can be significant uncertainty over whether conclusions obtained from the model are of a general nature or are specific to the functional forms chosen.

The generalised modelling approach (Gross and Feudel 2006; Kuehn et al 2013) permits precise mathematical statements about bifurcations of a dynamical system to be made in the presence of uncertainties about its precise form. Here, we use generalised modelling to make two types of inferences about a dynamical system. The first, and more traditional, use of generalised modelling will be to calculate the types of bifurcations that a generalised model can and is likely to undergo. Further below, we use generalised models together with time series data to generate early warning signals for regime shifts.

A generalised modelling bifurcation analysis proceeds as follows:

1. Write down a generalised model structure for the state variables and processes present in the system.

2. Symbolically calculate the Jacobian matrix of a fixed point in this generalised model.

3. Parameterise the Jacobian matrix directly using the so-called generalised parameters. Assign likely ranges of the generalised parameters based on knowledge of the system.

4. Calculate, using appropriate methods, the likely bifurcations to which these values of generalised parameters can lead.

This procedure will be described in further detail below with the aid of a simple example. We also note that the procedure described here above skips the normalisation step often used in previous generalised modelling studies (Gross and Feudel 2006; Kuehn et al 2013). This leads to the presence of an additional parameter in the Jacobian, which we call the 'steady-state ratio' below, but which has no effect on the bifurcation analysis.

Generalised modelling shares its mathematical basis, the bifurcations of dynamical systems, with both the qualitative theory of differential equations (Kelley and Peterson 2010) and catastrophe theory (Zeeman 1977). Indeed, generalised modelling could be considered a systematic way of parameterising a model before analysing its bifurcations with the tools of qualitative differential equation theory. Two key points of distinction of the generalised modelling are that: (i) it parameterises the Jacobian matrix directly, rather than the original functional forms; and (ii) it provides a systematic framework for connecting a generalised model structure to properties of real systems, through the generalised parameters (see below) or time series observations (in the case of a generalised modelling-based early warning signal). Generalised modelling therefore allows for the investigation of how processes, even incompletely characterised processes, lead to bifurcations 
of the system. Frequent failure to investigate how systemspecific processes could lead to the abstract, general geometries of catastrophes was a major contributor to the controversy over some strands of catastrophe theory (Guckenheimer 1978).

Conceptually, generalised modelling is also similar to systems dynamics approaches (Sterman 2000), structural equation modelling (Kline 2011) and flexible functional forms (Chambers 1988). A generalised model could be considered a causal loop diagram or stock and flow diagram from system dynamics in mathematical form, which we then further manipulate to obtain general mathematical results without resorting to simulating specific systems. Like generalised modelling, structural equation modelling (SEM) explores the consequences of linkages within a network of interacting variables; unlike generalised modelling, SEM can statistically test for the presence and strength of linkages, but does not commonly explore nonlinear dynamics and transitions of the variables. In economics, flexible functional forms are a general way to determine functional relationships directly from data, but they generally are not forms that are convenient for a subsequent bifurcation analysis.

To formulate a generalised model, the important state variables in the system and the processes through which they interact must first be identified. As a simple example, consider a single population $X$ (which may be of animals, of people, of people holding a particular opinion) that can increase due to a gain process $G(X)$ and decrease due to a loss process $L(X)$, both of which may depend on the current population $X$. A generalised model, in differential equation form, for the population $X$ is then

$\frac{\mathrm{d} X}{\mathrm{~d} t}=G(X)-L(X)$.

To investigate the bifurcations of this generalised model, we assume that the system has a fixed point, that is, some value $X^{*}$ where if $X(0)=X^{*}$ then $X(t)=$ $X^{*}$ for all $t>0$. Throughout this article we use the asterisk to denote a quantity evaluated at the fixed point. We then calculate the Jacobian matrix of the generalised model at the fixed point, and calculate the eigenvalues of the Jacobian, from which we can establish stability and bifurcations as described in Sec. 2.1. For Eq. (1), the Jacobian matrix $\mathbf{J}$ consists of a single element which is also the eigenvalue, $\lambda$,

$\mathbf{J}=\lambda=\left.\frac{\mathrm{d} G}{\mathrm{~d} X}\right|^{*}-\left.\frac{\mathrm{d} L}{\mathrm{~d} X}\right|^{*}$.

Therefore to determine the bifurcations of the system, we need to determine the possible ranges of these two derivatives.
To better relate the derivatives in the model to properties of a real-world system, we re-write Eq. (2) in a different form. We introduce:

- the scale parameter $\alpha=G^{*} / X^{*}$, which, with dimensions of inverse time, is a measure of the characteristic time scale of $X$.

- the dimensionless elasticity parameters

$$
G_{X}=\left.\frac{X^{*}}{G^{*}} \frac{\mathrm{d} G}{\mathrm{~d} X}\right|^{*} \quad \text { and } \quad L_{X}=\left.\frac{X^{*}}{L^{*}} \frac{\mathrm{d} L}{\mathrm{~d} X}\right|^{*} .
$$

The elasticity parameters give an indication of the non-linearity of the process near the fixed point. For example, a linear function $f(x)=a x$ has elasticity 1 for all $x$. A constant function $f(x)=a$ has elasticity 0 for all $x$.

The scale and elasticity parameters, and in more complicated examples another type of parameter called the ratio parameter, are collectively referred to as generalised parameters, as they are used to represent the dynamics of a general class of models rather than parameterising a particular model. Using these generalised parameters, Eq. (2) becomes

$\lambda=\alpha\left(G_{X}-L_{X}\right)$,

where we have also used the fact that $G^{*}=L^{*}$.

Next, we identify what conditions on the generalised parameters can give rise to different types of bifurcations. As described in the previous section, a zero eigenvalue of $\mathbf{J}$ indicates a fold bifurcation, while a pair of imaginary conjugate eigenvalues indicates a Hopf bifurcation. In this simple example, we can conclude that no Hopf bifurcations are possible (since there is only one eigenvalue, which is real) and, provided $\alpha>0$, a fold bifurcation can only occur when $G_{X}=L_{X}$.

The final step of the generalised modelling process is to make use of contextual knowledge about the processes to evaluate the likelihood of the identified bifurcations. In this example, suppose we know that the loss process $L(x)$ is approximately linear (elasticity 1 ), while the gain process $G(X)$ is linear at $X=0$ (elasticity 1 ) but saturates at high $X$ (elasticity 0$)$. Therefore $L_{X} \approx 1$ and $0<G_{X}<1$ for any non-zero population so a fold bifurcation is unlikely. If, however, $G(X)$ took a sigmoidal-type shape (with a quadratic or higher-order shape near $X=0$ ) then the elasticity $G_{X}$ could reach and exceed 1 and a fold bifurcation may be possible.

The generalised model that we analyse below is twodimensional. For this system, we search for bifurcations by identifying combinations of generalised parameters that satisfy

$\operatorname{det} \mathbf{J}=0$ 
for fold bifurcations and

$\operatorname{tr} \mathbf{J}=0$ and $\operatorname{det} \mathbf{J}>0$

for Hopf bifurcations, where tr denotes the trace of the matrix. ${ }^{2}$ We also test whether $\operatorname{det} \mathbf{J}$ (for fold bifurcations) or $\operatorname{tr} \mathbf{J}$ (for Hopf bifurcations) changes sign at the candidate bifurcation points, as is necessary for a true bifurcation.

\subsection{Early warning signals}

Consider a dynamical system that is undergoing noisy fluctuations due to a fast noise forcing. As the dynamical system approaches a fold bifurcation the standard deviation of these fluctuations, under a linear approximation, diverges to infinity and their autocorrelation increases towards one (Scheffer et al 2009; Kuehn 2013). Although the linear approximation will not hold arbitrarily close to a fold bifurcation, increasing variance and autocorrelation are two well-known early warning signals that have both been shown to precede regime shifts in simulation studies, in laboratory and field experiments and in data of past regime shifts (Lenton 2012; Scheffer et al 2012).

We examine whether variance and autocorrelation early warning signals also precede the regime shifts we explore in our social-ecological system. To calculate the early warning signals we use the R package 'earlywarnings' (Dakos et al 2012a) developed by Dakos et al (2012b). A bandwidth of 20 for the detrending of the time series was used.

We also explore a third early warning signal, the recently developed generalised modelling-based early warning signal (Lade and Gross 2012). Unlike the variance and autocorrelation approaches, which rely purely on time series data of the system, this approach also makes use of structural knowledge about the system. As the name suggests, the approach begins by constructing a generalised model and then formally calculating its Jacobian matrix as described in Sec. 2.2. Instead of assigning ranges to the elements of the Jacobian matrix through generalised parameters, time series observations of the state variables and processes are used to directly estimate the derivatives in the Jacobian matrix. The particular computations used to estimate the derivatives depend on the structure of the generalised

2 These conditions can be easily derived by noting that the eigenvalues of a two-dimensional Jacobian matrix are $\lambda=\frac{1}{2} \operatorname{tr} \mathbf{J} \pm \frac{1}{2} \sqrt{\operatorname{tr}^{2} \mathbf{J}-4 \operatorname{det} \mathbf{J}}$. We emphasise that Eq. (4) is only valid in two dimensions; approaches that also work for higher dimensions include the Routh-Hurwitz criteria and the method of resultants (Gross and Feudel 2004). model and on the available data; we will derive an algorithm appropriate to the model studied here.

\subsection{Social ostracism and resource model}

We use the tools described above to analyze regime shifts in a stylised social-ecological model. Based on the TSL model, we consider a resource, of resource level $R$, that is being harvested by a community of users. A proportion $f_{c}$ of the harvesters co-operate to harvest at a socially optimal level while the remaining harvesters 'defect' and harvest at a higher level out of self-interest.

We write the resource dynamics in generalised form as

$\frac{\mathrm{d} R}{\mathrm{~d} t}=c-D(R)-Q\left(E\left(f_{c}\right), R\right)$,

where $c$ is the resource inflow or growth rate (and is independent of the current resource level), $D(R)$ is the natural resource outflow rate or mortality, and $Q(E, R)$ is the resource extraction. Here $E\left(f_{c}\right)$ is the total effort exerted by the harvesters, which decreases with increasing proportion of co-operators. In practice, the resource could be fish in a fishery, water in an irrigation system, an unpolluted atmosphere, etc.

We, like TSL, use the replicator dynamics of evolutionary game theory to model the dynamics of the fraction of co-operators $f_{c}$. In its most general form, the replicator equation for two strategies is $\mathrm{d} f_{c} / \mathrm{d} t=$ $f_{c}\left(1-f_{c}\right)\left(U_{c}-U_{d}\right)$, where $U_{c}$ and $U_{d}$ are the utilities for a co-operator and a defector, respectively. In our model, the utilities of defectors and co-operators can differ in three ways.

First, the income received by defectors and co-operators differs due to their harvesting activities, by an amount we denote by $F(E, R)>0$ that depends on the current resource level $R$ and the total effort $E$. Second, the costs incurred by defectors and co-operators differ due to their harvesting activities by an amount $W>0$. We expect both income and costs to be higher for defectors than for co-operators, since defectors invest more effort.

The income difference minus the cost difference constitutes the payoff difference between defectors and cooperators. Like TSL, we include a third difference between co-operator and defector utility, that the defectors can be socially ostracised by the co-operators, to reduce the utility of the larger payoff that they would otherwise obtain. We denote by $\omega\left(f_{c}\right)>0$ the degree to which ostracism reduces a defector's utility, with the property that the size of this reduction is expected to increase with increasing $f_{c}$. Ostracism can occur with the 
presence of individuals in the community with otherregarding preferences (Fehr and Fischbacher 2002) and when fear of community disapproval leads to pressure to conform with the social norm (Cialdini and Goldstein 2004).

We note that the social ostracism modelled by TSL, which we follow in generalised form here, is non-costly, in that it does not cost the co-operators to impose this punishment. Non-costly ostracism may occur when the community builds upon available social capital (Bowles and Gintis 2002) to deny defectors important services, such as refusing to loan machinery or refusing transportation to market (Tarui et al 2008; Tavoni et al 2012). Indeed, sanctioning can even provide benefits to the enforcer (Ostrom 1990). Other models in which cooperation is encouraged in a non-costly manner include those of Osés-Eraso and Viladrich-Grau (2007), Iwasa et al (2007) and Tarui et al (2008).

These three differences between co-operator and defector utility result in the following equation for changes in the fraction of co-operators,

$\frac{\mathrm{d} f_{c}}{\mathrm{~d} t}=f_{c}\left(1-f_{c}\right)\left(-F\left(E\left(f_{c}\right), R\right)+W+\omega\left(f_{c}\right)\right)$.

As described in the Supporting Text (Section 1), some of our definitions differ from those originally used by TSL. These changes were made in order to reduce Eq. 6 to a number of unknown functions and parameters that is as small as possible while still clearly representing the processes at work in the social-ecological system.

Eqs. (5-6) constitute our generalised model of social ostracism and resource dynamics, hereafter referred to as 'our social-ecological model'. We denote by the ecological or social subsystem the relevant part of the system with any feedback from the other part set to a constant value. It is clear that there are two key processes that link the ecological and social subsystems: extraction of the resource $Q$, and the income (difference) $F$ gained by harvesters due to extraction (Fig. 2). For both these processes, the effect of the social subsystem is mediated by the total effort $E$ of the harvesting community.

To illustrate or to test our generalised modelling we will also produce time series data using simulation models. For this purpose we will use the original TSL model as described in the Supporting Text (section 1), and modifications thereof as described below. The TSL model is a specific case of our generalised model; the precise correspondence is shown in the Supporting Text.

We also use simulations of the TSL model, with added process noise, to test the early warning signals. On $R$ the noise is purely additive; for $f_{c}$ the appropriate Itō noise term has variance proportional to $f_{c}\left(1-f_{c}\right)$

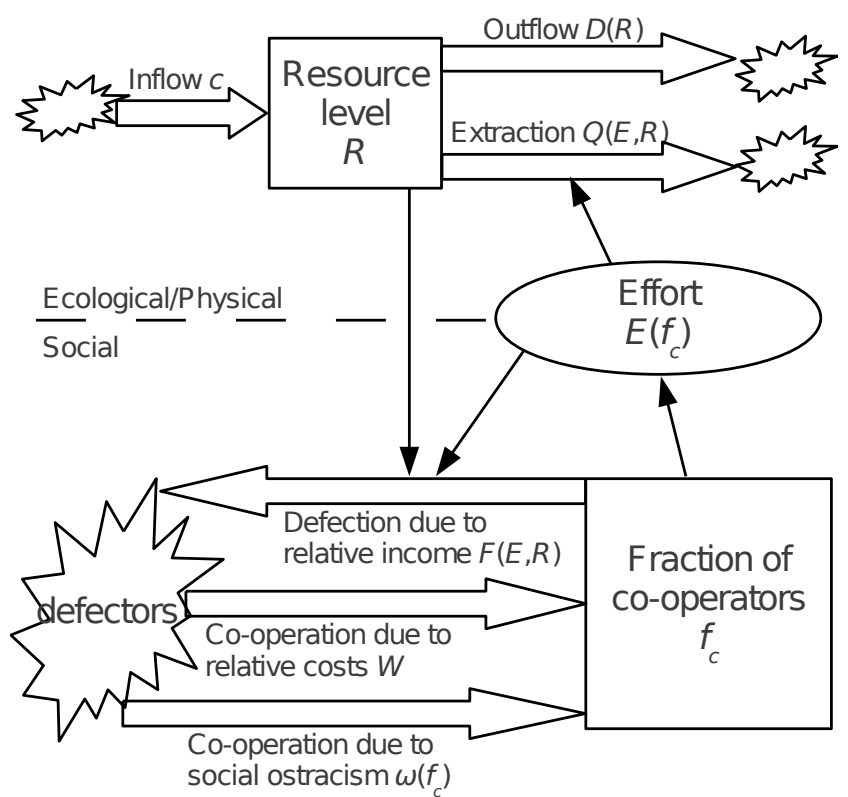

Fig. 2 Schematic of the generalised model Eqs. (5-6). Employing some of the systems dynamics conventions (Sterman 2000), we represent flows by double-line arrows, influences by single-line arrows, state variables by rectangles, intermediate quantities by ovals, and sources and sinks of flows with explosion symbols.

(Traulsen et al 2005). During the simulation, which lasted from $t=0$ to 500, we varied the TSL parameters $c$ and $w$ (see Supporting Text) according to $c(t)=$ $40+0.024 t$ and $w(t)=18-0.08 t$. The results of the simulation were sampled at intervals of $\Delta t=1$ time unit.

\section{Results}

\subsection{Generalised modelling bifurcation analysis}

Following the generalised modelling procedure, let there be a fixed point $\left(R, f_{c}\right)=\left(R^{*}, f_{c}^{*}\right)$ where $d R / d t=$ $d f_{c} / d t=0$. Furthermore, we concentrate on mixedstrategy fixed points, $0<f_{c}<1$. Pure strategy $\left(f_{c}=0\right.$ or 1) fixed points also (indeed, always) exist, but we will see below (Fig. 4) that it is usually the bifurcations of the mixed-strategy fixed points that give rise to regime shifts. The Jacobian matrix of the generalised model in Eqs. (5-6) evaluated at this fixed point is

$$
\mathbf{J}=\left[\begin{array}{c}
-\alpha_{R} \beta_{R} D_{R}-\alpha_{R}\left(1-\beta_{R}\right) Q_{R} \\
-\alpha_{f} F_{R} / \delta_{f c}^{R}
\end{array} \quad \begin{array}{c}
-\alpha_{R}\left(1-\beta_{R}\right) Q_{E} E_{f c} \delta_{f c}^{R} \\
\quad-\alpha_{f} F_{E} E_{f c}+\alpha_{f}\left(1-\beta_{F}\right) \omega_{f c}
\end{array}\right]
$$

with the generalised parameters as defined in Table 1. 
Table 1 Definitions and interpretations of the generalised parameters used in the bifurcation analysis. Values of the generalised parameters that correspond to the TSL model are indicated, as are other values used in the bifurcation analysis. For definitions of TSL's $k, e_{d}, e_{c}, a, b$, see the Supporting Text.

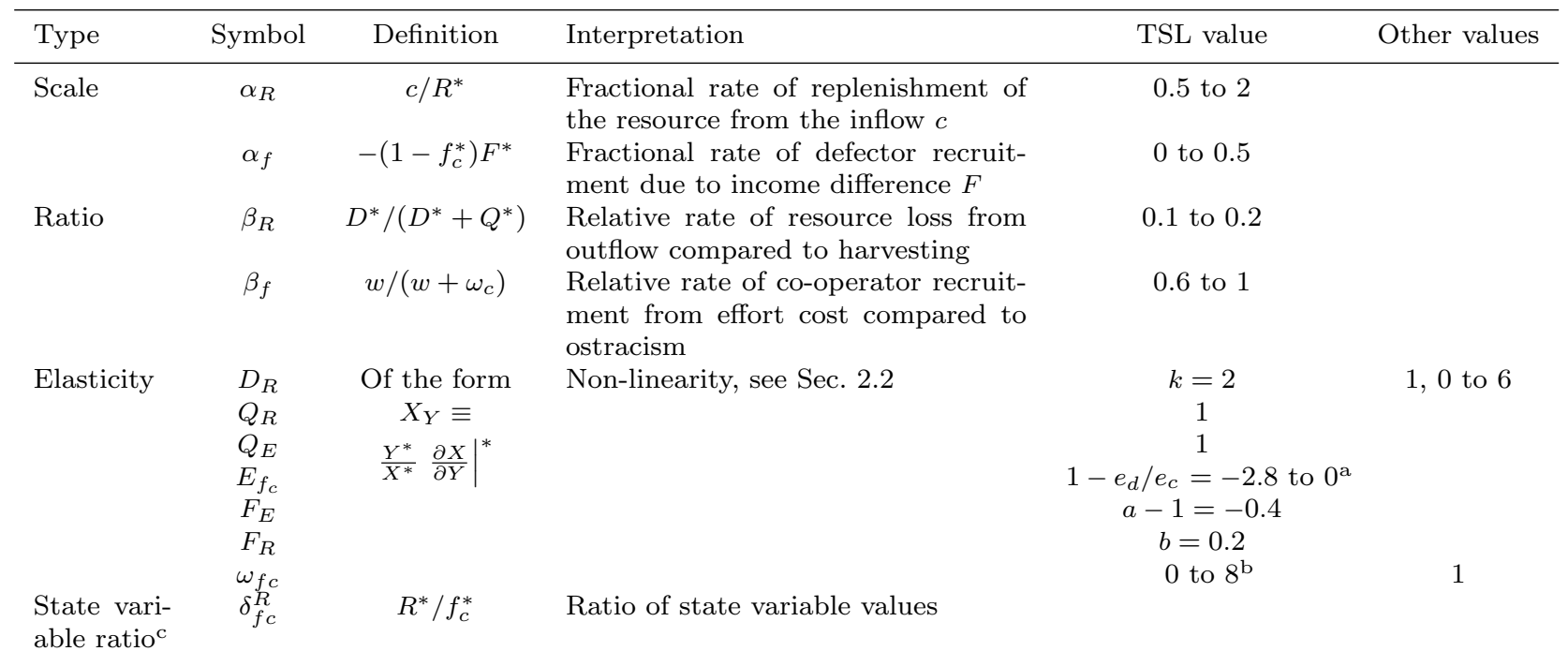

a The effort elasticity is negative because an increase in co-operators decreases the effort. The elasticity is zero under full defection $\left(f_{c}=0\right)$, because a large fractional change in the number of co-operators leads to a small fractional change in the total effort. In the TSL model, the elasticity takes its largest negative value at full co-operation $\left(f_{c}=1\right)$.

b The particular ostracism function chosen by TSL causes the elasticity $\omega_{f c}$ to reach extremely high values at values of $f_{c}$ where the magnitude of the function $\omega\left(f_{c}\right)$ itself is very small. Another function that reproduces TSL's $\omega\left(f_{c}\right)$ very closely is $\omega\left(f_{c}\right)=0.34 f_{c}^{8} /\left(0.55^{8}+f_{c}^{8}\right)$, which has the more reasonable range of elasticities indicated above.

c The state variable ratio $\delta_{f c}^{R}$ is not required in any of the following calculations.

Using $\mathbf{J}$ and the condition in Eq. (3), we find that for generalised parameters corresponding to the TSL model (Table 1), fold bifurcations are widespread (Fig. 3). Specifically, for any combination of $\omega_{f c}$ and $D_{R}$ in the range plotted, there is an $E_{f c}$ that leads to a fold bifurcation. This matches the bifurcations observed by TSL both in their previous work, as indicated by the appearance and disappearance of their mixed equilibrium states, as well as in an upcoming study (Schlüter et al in prep.).

Although the Jacobian $\mathbf{J}$ does permit Hopf bifurcations for some extreme parameter combinations, the Hopf conditions [Eq. (4)] are not simultaneously satisfied anywhere in the generalised parameter space plotted in Fig. 3. We conclude that Hopf bifurcations, and consequently oscillatory states, are unlikely to be observed for models similar to the TSL model.

If we ignore the social dynamics in this system and set the total effort $E$ to a constant, the eigenvalue at a fixed point of $R$ in the ecological subsystem is

$\lambda_{\text {ecol }}=-\alpha_{R} \beta_{R} D_{R}-\alpha_{R}\left(1-\beta_{R}\right) Q_{R}$.

Provided that $D_{R}$ and $Q_{R}$ are always positive (as they are in the TSL model), this eigenvalue is always negative. Therefore, in this model, no bifurcation can occur and in particular no regime shift can occur. It is clear

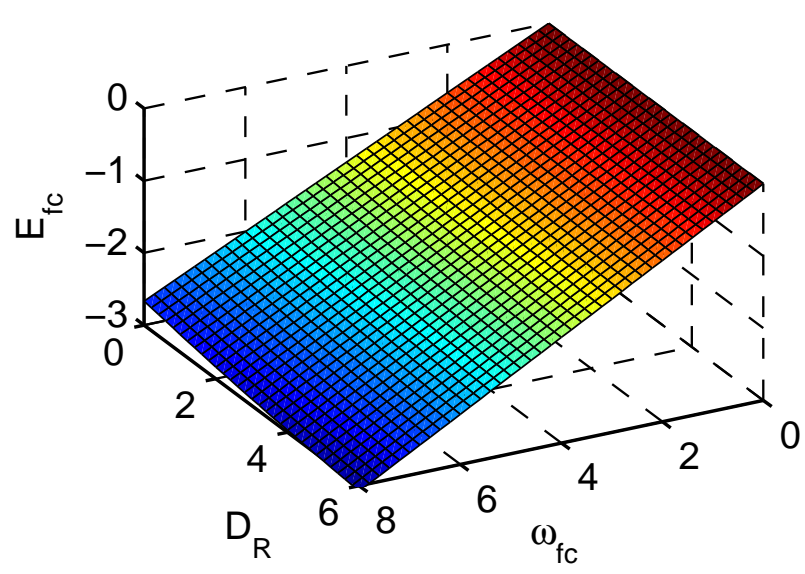

Fig. 3 Generalised modelling analysis. Surface of fold bifurcations for ranges of generalised parameters matching the TSL model.

that by ignoring the social dynamics it is impossible to appropriately model the regime shift that can occur in our social-ecological system.

To obtain a regime shift in the ecological subsystem alone, $D_{R}$ or $Q_{R}$ would have to be sufficiently negative for $\lambda_{\text {ecol }}$ to reach zero. In traditional models of ecological regime shifts, this is achieved because $D(R)$ (or inflow rate, which here is a constant $c$ ) is sufficiently nonlinear. There are of course many ecological and physical 
systems with such a regime shift-inducing non-linearity (Scheffer et al 2001). Our purpose here is to show that regime shifts of ecological states can occur even if the ecological subsystem alone does not have a regime shift.

Returning to Fig. 3 we note, in addition to the ubiquity of fold bifurcations, that (i) fold bifurcations are possible for a large range of $\omega_{f_{c}}$, including values near 1 , and (ii) that the presence of fold bifurcations is not strongly affected by the value of $D_{R}$. This indicates that close to linear ostracism and resource outflow functions $\omega\left(f_{c}\right)$ and $D(R)$ may be sufficient to produce a fold bifurcation. We confirm this prediction below using a simulation model.

Furthermore, setting $\omega\left(f_{c}\right) \propto f_{c}$ and $D(R) \propto R$ removes all non-linearity from both the purely social and purely ecological components of the dynamics. The only remaining non-linearities are contained in the linkage between the social and ecological subsystems. This linkage is comprised by the processes $Q(E, R)$, which specifies the amount of resource extracted by the harvesters, and the income difference $F(E, R)$, which specifies the effect of resource extraction on the fraction of co-operators. ${ }^{3}$ Thus as well as arising from non-linearities in the ecological or social dynamics, regime shifts can also arise from non-linearities in the linkages between them.

\subsection{Bifurcations of simulation models}

We next tested the general predictions of the generalised modelling analysis above with simulations of the TSL model, and variants thereof.

Beginning with the parameter set used by TSL (see Supporting Text, Section 1), changing the resource inflow readily triggered a fold bifurcation (Fig. 4a). In fact, changes in any of many different drivers, including effort cost (Fig. 4e), the strength of ostracism (Fig. $4 \mathrm{f}$ ), or even multiple drivers changing simultaneously (resource inflow and effort cost, Fig. 4g), could trigger the fold bifurcation. We conclude that, as predicted by the generalised modelling analysis, fold bifurcations and therefore regime shifts are easy triggered in our social-ecological model.

Somewhat counter-intuitively, the regime shift from a high co-operation state that led to breakdown of the social norm and collapse of the resource was triggered by an increasing resource inflow, countering the common understanding that it is scarcity that leads to conflict. In this model, the regime shift occurred due to an initially increasing resource level that led to a greater

3 In the TSL model, these linkages have the following nonlinearities: $Q(E, R) \propto E R$ and $F(E, R) \propto E^{a-1} R^{b}$. increase in the defector income than in co-operator income, due to allocation of net production according to effort. Increased defection then decreased the effectiveness of social ostracism and also increased extraction of the resource, culminating in a collapse in co-operation and in resource levels.

The bifurcation remained (Fig. 4b) when we used a variant of the TSL model with $\omega\left(f_{c}\right) \propto f_{c}$ and $D(R) \propto$ $R$ (Supporting Text, section 1). The size of the regime shift was not as large as in the case of strongly nonlinear $\omega\left(f_{c}\right)$ (Fig. 4a), however. We conclude that, as predicted by the generalised modelling analysis, the presence of the regime shift is robust to the functional form of the ostracism process $\omega\left(f_{c}\right)$, and also to the functional form of the resource outflow $D(R)$. As predicted by the generalised modelling analysis, a simulation model of the ecological subsystem alone, however, did not have a bifurcation (Fig. 4h).

Although the ecological subsystem alone does not display a regime shift, the consequences of the socialecological regime shift can be just as serious for the resource levels as a purely ecological shift. The regime shift associated with increasing resource inflow (Fig. 4a) led to a significant drop in resource levels (Fig. 4c). The total payoff (income minus costs) that the community received also collapsed (Fig. 4d). In this state, social ostracism is largely ineffective due to the small population of co-operators. Re-establishing the ostracism norm and the associated high resource state would in this model require a large drop in resource inflow, or may even be impossible in the absence other mechanisms to re-establish the norm.

\subsection{Early warning signals}

A good standard deviation or autocorrelation warning signal should display a clear upwards trend well in advance of the critical transition. In the early warning literature, these trends in indicators are often quantified with the Kendall- $\tau$ statistic (Dakos et al 2012b). We applied the standard early warning suite to time series of both $R$ and $f_{c}$ leading up to the transition (Fig. 5). We observed, for the simulation dataset used (Fig. 5, first row), weak trends in autocorrelation and stronger trends in standard deviation (largest Kendall $\tau$ statistic 0.86 for standard deviation of residuals of $f_{c}$ ).

We next constructed a generalised modelling-based early warning signal. We assumed that the following quantities could be measured:

- The resource level, $R$

- The fraction of co-operators, $f_{c}$ 

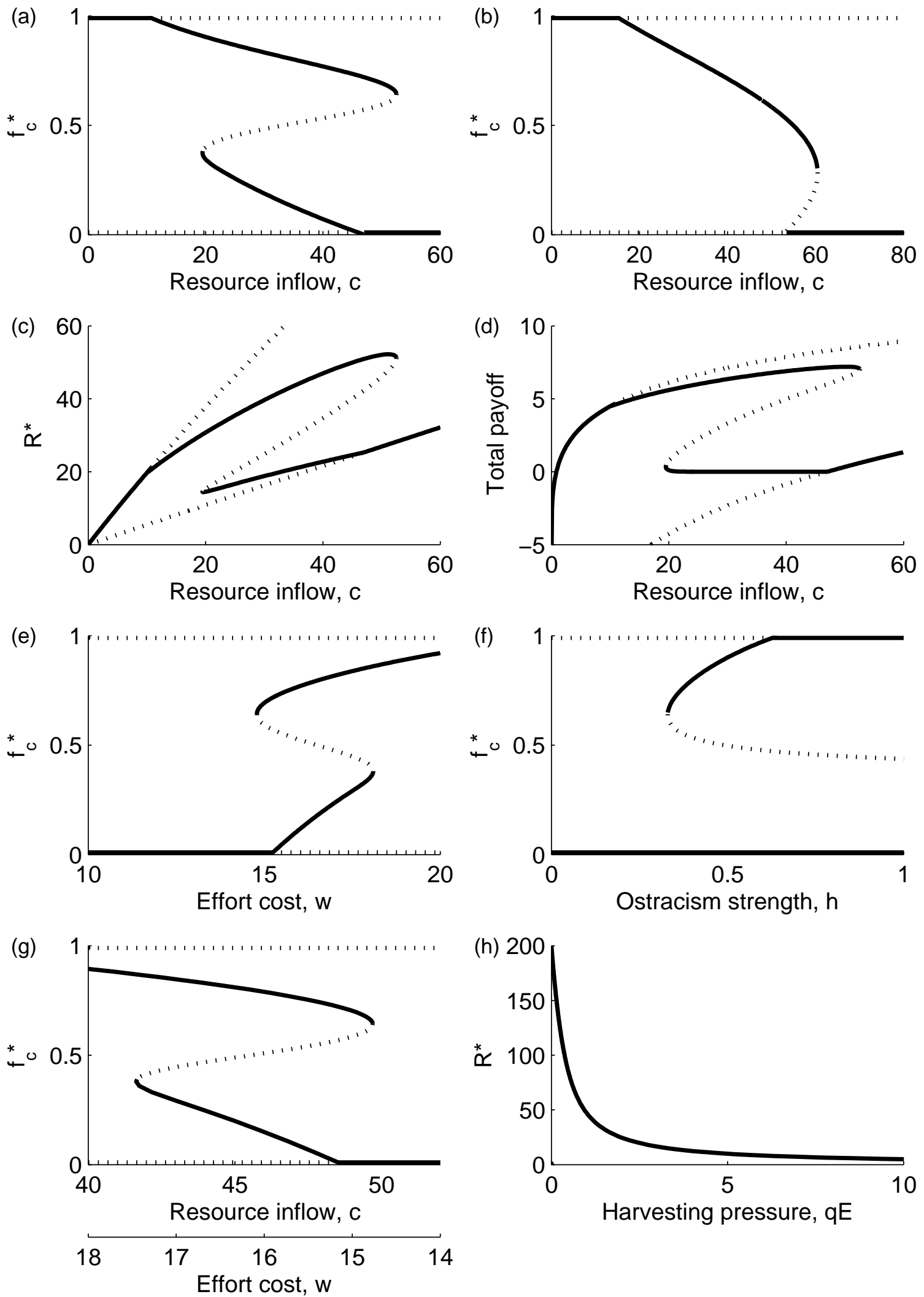

Fig. 4 Bifurcation diagrams of simulation models. The fraction of co-operators $f_{c}$ are plotted for the fixed points of the TSL model (Supporting Text, Section 1) with respect to changes in: (a) the resource inflow; (b) the resource inflow with the functions $\omega_{f c}$ and $D(R)$ set to linear forms (see Supporting Text, Section 1); (e) the cost of harvesting effort; (f) the strength parameter of the ostracism function; ( $\mathrm{g}$ ) both resource inflow and effort cost at the same time. (h) Fixed points $R$ of the isolated ecological subsystem (Supporting Text, Section 1). In (c) the resource levels and in (d) the total community payoff $n\left[f_{c}^{*} e_{c}+\left(1-f_{c}^{*}\right) e_{d}\right]\left[f\left(E^{*}, R^{*}\right) / E^{*}-w\right]$ (see Supporting Text, Section 1 for definitions of symbols) corresponding to the fixed points in (a) are shown. Solid lines denote stable fixed points, dotted lines denote unstable fixed points. 
$\mathbf{R}$

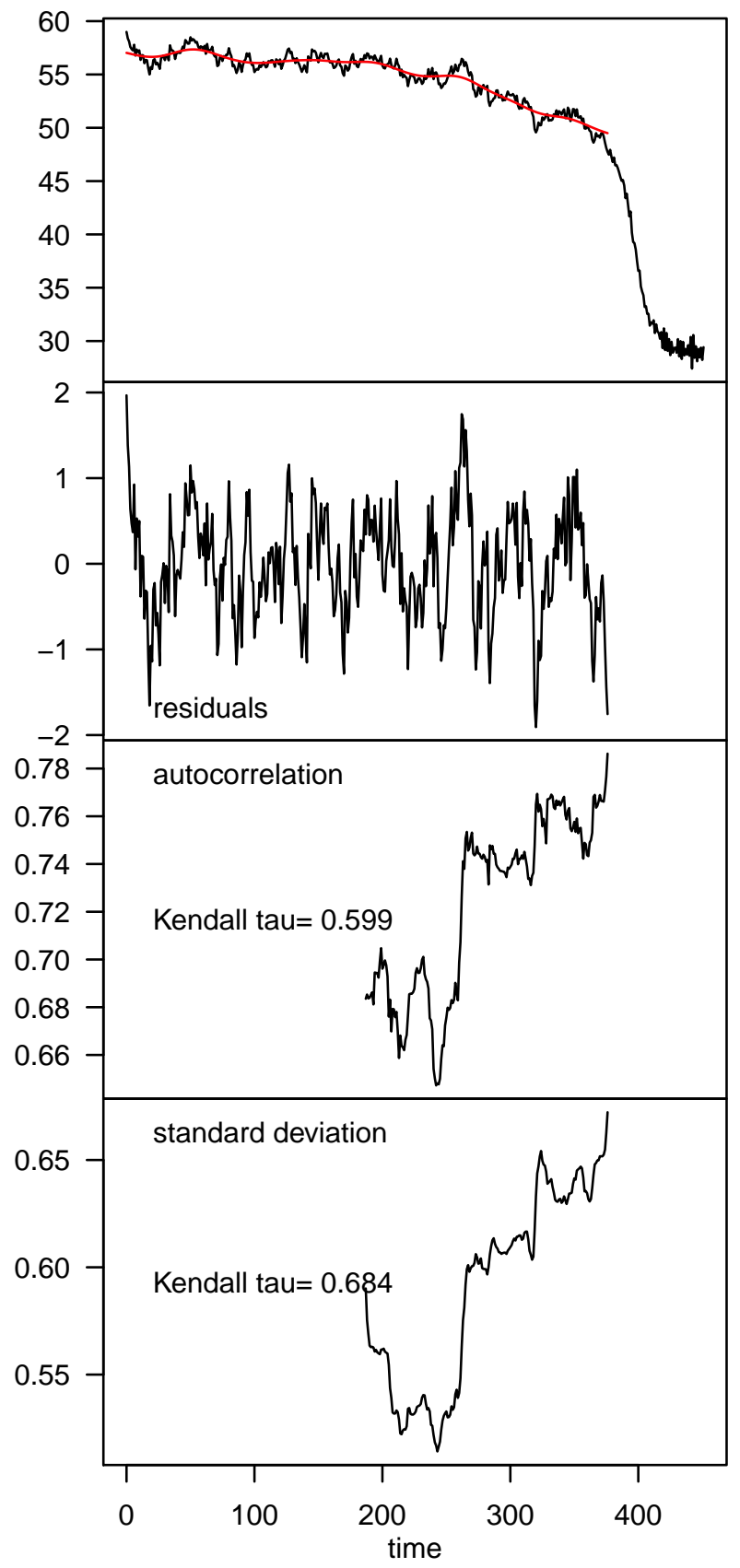

$\mathrm{f}_{\mathrm{c}}$

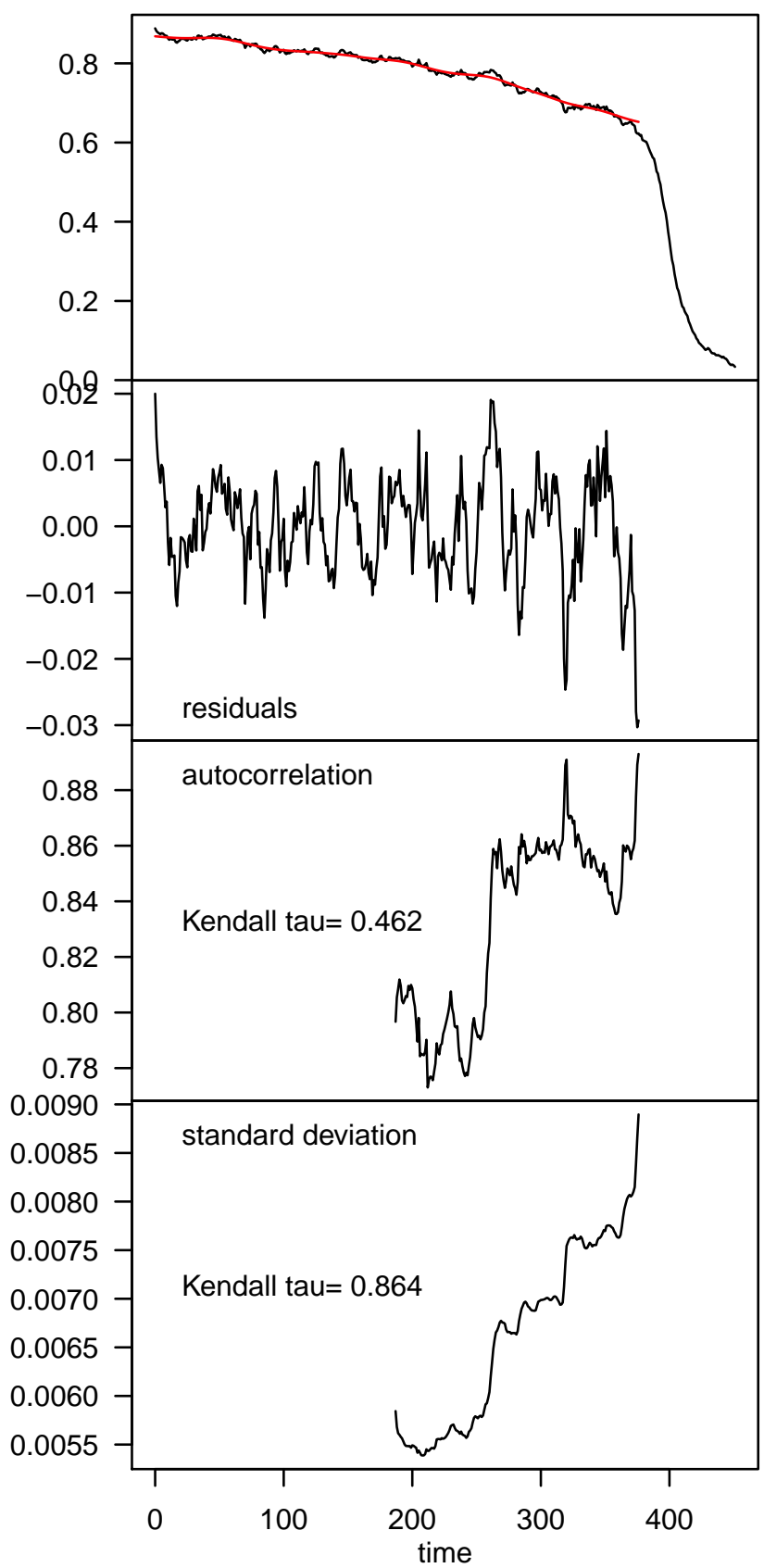

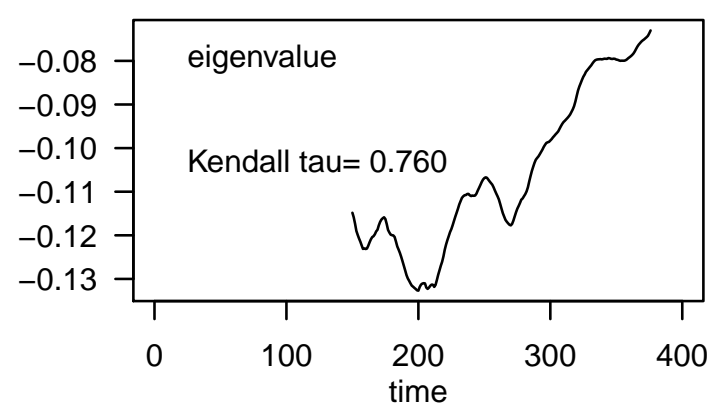

Fig. 5 Early warning signals. (top) Time series with filtered fit, detrended fluctuations, and autocorrelation and standard deviation of the detrended fluctuations for $R$ and $f_{c}$, respectively, in the lead-up to the regime shift. (bottom) Generalised modelling-based early warning signal preceding the regime shift. 
- The resource outflow, $D$ (for example, natural fish mortality or natural water losses due to evaporation or outflow). Could be replaced by observations of resource inflow $c$ if more easily measured.

- The total resource extraction, $Q$ (for example, total fish caught or total water used for irrigation)

- The income difference between a defector and a cooperator, $F$.

- The cost difference between a defector and a cooperator, $W$.

We assumed that the regime shift was being triggered by changes in resource inflow $c$ and/or effort costs $W$. To complete the generalised modelling analysis we also required the assumptions that: resource extraction is linear in the resource level, $Q \propto R$; and income difference is sub-linear in resource level with known elasticity $b, F \propto R^{b}$. We found however that the results of the generalised modelling early warning signal are not sensitive to the value of $b$ used. In the following, we used $b=0.5$, significantly different to the elasticity actually used in the simulation $(b=0.2)$.

Following the approach outlined by Lade and Gross (2012), we derived an algorithm to calculate the eigenvalues of the generalised model from the quantities in the above list (Supporting Text, Section 2). Key outputs of this algorithm included the derivatives of the income difference $F$, resource extraction $Q$ and ostracism $\omega$ with respect to the fraction of co-operators $f_{c}$.

From the TSL model with changing parameters described at the end of Section 2.4, we generated time series for the list of quantities above. We then applied our algorithm, which yielded time series of two eigenvalues. A clear warning signal would be a negative (stable) eigenvalue increasing consistently towards the stability boundary of zero eigenvalue. One of the eigenvalues we calculated was always stable and far from the stability boundary. The other eigenvalue, however, displayed a clear increasing trend (Fig. 5, Kendall tau statistic $0.760)$.

On the basis of these results, we find standard deviation and generalised modelling eigenvalue to be good candidates for early warning signals for regime shifts in this social-ecological system. However we emphasise that these are preliminary results. A more thorough analysis would explore the sensitivity of the observed trends to different algorithm parameters such as smoothing or detrending constants and rolling window size (Dakos et al 2012b), a consideration of false alarm and missed detection rates through receiver-operator characteristics (Boettiger and Hastings 2012), as well as repetitions over an ensemble of realisations of the noise.
The early warning signal approaches described here also have differing demands on the amount and type of data and knowledge required. The autocorrelation and standard deviation approaches require only highfrequency observations of a single quantity. The generalised modelling-based warning signal, in contrast, requires knowledge of the structure of the social-ecological system as well as regular observations of all state variables and several of the processes by which they interact. It is hoped that, for some regime shifts, such additional, system-specific information will improve the reliability of the warning signal, as well as decreasing the frequency at which time series need to be sampled (Lade and Gross 2012; Boettiger and Hastings 2013).

The generalised modelling approach for early warning signals is also itself in an early stage of development. Future improvements could include statistical approaches: to incorporate partial knowledge about derivatives in the Jacobian matrix; similar to the approach of Boettiger and Hastings (2012), to test the fit of alternative generalised models; and to calculate the level of confidence in an early warning trend.

\section{Discussion and implications for management}

In the social-ecological system studied here, the ecological subsystem could not by itself undergo a regime shift at all, whereas regime shifts in the social-ecological system were common. The results of the social-ecological regime shift were as dramatic as purely ecological regime shifts that occur when the human impact acts as a simple driver: there was a rapid, large and persistent collapse of the resource, along with an associated collapse of the social norm and community payoffs. We conclude that failing to model a natural resource as a socialecological system, which requires including the dynamics of human (and institutional) behaviour, can lead to severely underestimating the potential for regime shifts. In a related result, undesirable regime shifts were previously shown to be avoidable if multiple feedbacks from the state of a complex ecological system were incorporated into management planning (Horan et al 2011).

The generalised modelling analysis showed the regime shift persists under variations to the shape of the ostracism function and the natural resource outflows. A regime shift even occurred when the social and ecological subsystems were completely linear, with nonlinearities arising only in the extraction and production processes that link the two subsystems. These findings have two implications for natural resource management. First, careful attention should be paid to the links between natural resources and human actors, for example, the way ecosystem services are used and contribute to 
human well-being. The link between natural resources and human well-being are in particular not well studied. Second, social-ecological systems that under current conditions are managed sustainably through high levels of cooperation (such as the Maine lobster fishery, Acheson and Gardner 2011) could potentially easily be destabilised by small changes in or increasing variability of important processes. This becomes particularly relevant in the context of global change, where resource dynamics are expected to become more variable or more extreme, thus potentially pushing a successful system rapidly into an unsustainable state.

Indeed, the ease with which a regime shift can be triggered lends support to a precautionary-like approach to managing social-ecological systems (Raffensperger and Tickner 1999): assume the system can undergo regime shifts, unless there is evidence otherwise. Such a precautionary approach may imply, for example: holding stocks at higher levels (Polasky et al 2011); using generic principles for increasing resilience (Biggs et al $2012 \mathrm{~b}$ ), such as engaging in a process of adaptive governance; or preparing to mitigate the effects of the regime shift if it is unlikely to be avoided (Crépin et al 2012).

Examining regime shifts demands viewing the socialecological system as a complex adaptive system (Levin et al 2012). As well as the possibility of non-equilibrium behaviour (such as regime shifts), an important complex adaptive property of the system we studied here is the ability of human actors to switch their harvesting strategies in response to changing resource levels. The tool of generalised modelling that we have used combines a complex adaptive systems view of the socialecological system with a precise mathematical setting and the ability to obtain results in the presence of uncertainty about specific forms of interactions. Given the high degree of uncertainty often associated with the detailed workings of social and ecological processes, we anticipate generalised modelling to be a useful tool in future work on social-ecological systems. Although recently developed, generalised modelling (sometimes also called structural kinetic modelling) has already yielded successes in ecology (Gross et al 2009; Aufderheide et al 2012), physiology (Zumsande et al 2011) and molecular biology (Steuer et al 2006; Gehrmann and Drossel 2010; Zumsande and Gross 2010), where the details of specific interactions can likewise be difficult to determine.

We also studied regime shifts in our social-ecological system using a simulation model. The regime shifts could be triggered by many different social and ecological drivers, and also a combination of drivers. We also obtained the result, on first glance counterintuitive, that increasing the resource inflow led to a collapse of co-operation and sudden decrease in the resource level and payoffs, due to the defectors gaining more from an increase in resource level than co-operators. We conclude that sometimes not only can the regime shift itself be surprising, but the direction of change in a driver that triggers a regime shift can also surprise.

Given the widespread existence and sometimes surprising nature of these social-ecological regime shifts, some early warning of an impending regime shift would be highly desirable, in order to avoid or at least mitigate the effects of the regime shift. We tested the performance of standard early warning signals for one of the regime shifts produced by the TSL model. The autocorrelation warning signal showed only a weak indication of the transition, with standard deviation (particularly of $f_{c}$ ) and the generalised modelling-based signal showing stronger signals. In practice, the effectiveness of an early warning signal can depend on a number of factors, including: the magnitude of the noise (Contamin and Ellison 2009; Perretti and Munch 2012); appropriate choice of variable(s) to observe; whether a potential associated with the dynamics exists and is smooth (Hastings and Wysham 2010); the rate at which the driver is changing relative to the inherent time scales of the systems, such as life spans (Bestelmeyer et al 2011); the observation rate compared to these inherent time scales (Bestelmeyer et al 2011); non-stationary noise statistics (Dakos et al 2012c); and indeed whether the regime shift is driven at all or is instead triggered by noisy fluctuations (Ditlevsen and Johnsen 2010). The autocorrelation, standard deviation and generalised modelling early warning approaches also have different requirements for the amount and type of data and knowledge required (Sec. 3.3). We conclude that early warning signal approaches show potential for warning of social-ecological regime shifts, which could be valuable in natural resource management to guide management responses to variable and changing resource levels or changes in resource users. However, investigation of specific cases of social-ecological regime shifts is required to ascertain, first, the availability of the required data in those cases and, second, the robustness of the resulting early warning signals.

A third and very important criterion by which to evaluate an early warning signal in a specific case study is whether the signal can give sufficiently early warning for the transition to be avoided. Successfully averting a transition depends on a number of case-specific factors, including which drivers can be manipulated (Biggs et al 2009), the rate at which this can be done (Biggs et al 2009), how fast the system responds to a change in management (Contamin and Ellison 2009), and, importantly, how fast the uncontrolled driver is 
itself changing. In the limit of a very slowly changing driver, for example, warning signals are likely to provide sufficient notice for action to be taken, while in the limit of a very quickly changing driver, effectively an unpredictable shock, no warning signal could be sufficiently fast. Including management responses in the social-ecological system and evaluating whether warning signals can give sufficient notice for management actions to avert a regime shift are beyond the scope of the stylised models used here. A thorough investigation would require more detailed mechanistic models as well as data pertaining to actual social-ecological regime shifts (Schlüter et al 2012b).

\section{Conclusions}

We studied stylised models of a social-ecological system of broad relevance: a common-pool resource, which is being harvested, and for which a normative mechanism amongst harvesters (social ostracism) encourages a socially optimal harvesting strategy. We found that neglecting the dynamics of the social subsystem of the social-ecological system led to models missing the existence of regime shifts in the system, regime shifts which could be as persistent and economically detrimental as a purely ecological regime shift. Furthermore, we found the regime shift to be robust to uncertainty about the specific shapes of the interaction processes, leading us to support the inclusion of regime shifts within a precautionary approach to managing ecosystems. Finally, we also showed that the early warning signals developed for ecological or physical systems may also be useful for anticipating regime shifts in social-ecological systems.

Acknowledgements The authors would like to thank Ralf Eichhorn, Thilo Gross, Jamila Haider, Juan Carlos Rocha and Nanda Wijermans for helpful comments on the manuscript. The research leading to these results has received funding from the European Research Council under the European Unions Seventh Framework Programme (FP/2007-2013)/ERC grant agreement no. 283950 SES-LINK. A.T. is supported by the Centre for Climate Change Economics and Policy, which is funded by the UK Economic and Social Research Council and Munich Re.

\section{References}

Acheson J, Gardner R (2011) The evolution of the Maine lobster V-notch practice: Cooperation in a prisoner's dilemma game. Ecology and Society 16:41

Aufderheide H, Rudolf L, Gross T (2012) Mesoscale symmetries explain dynamical equivalence of food webs. New Journal of Physics 14(10):105014

Bestelmeyer BT, Ellison AM, Fraser WR, Gorman KB, Holbrook SJ, Laney CM, Ohman MD, Peters DPC, Pillsbury
FC, Rassweiler A, Schmitt RJ, Sharma S (2011) Analysis of abrupt transitions in ecological systems. Ecosphere 2:129

Biggs R, Carpenter SR, Brock WA (2009) Turning back from the brink: Detecting an impending regime shift in time to avert it. Proc Natl Acad Sci USA 106(3):826-831

Biggs R, Blenckner T, Folke C, Gordon L, Norström A, Nyström M, Peterson G (2012a) Regime shifts. In: Hastings A, Gross LJ (eds) Encyclopedia of Theoretical Ecology, University of California Press, pp 609-617

Biggs R, Schlüter M, Biggs D, Bohensky EL, BurnSilver S, Cundill G, Dakos V, Daw TM, Evans LS, Kotschy K, Leitch AM, Meek C, Quinlan A, Raudsepp-Hearne C, Robards MD, Schoon ML, Schultz L, West PC (2012b) Toward principles for enhancing the resilience of ecosystem services. Annual Review of Environment and Resources $37: 421-448$

Boettiger C, Hastings A (2012) Quantifying limits to detection of early warning for critical transitions. Journal of The Royal Society Interface

Boettiger C, Hastings A (2013) From patterns to predictions. Nature 493:157-158

Bowles S, Gintis H (2002) Social capital and community governance. The Economic Journal 112(483):F419-F436

Carpenter SR, Mooney HA, Agard J, Capistrano D, DeFries RS, Daz S, Dietz T, Duraiappah AK, Oteng-Yeboah A, Pereira HM, Perrings C, Reid WV, Sarukhan J, Scholes RJ, Whyte A (2009) Science for managing ecosystem services: Beyond the Millennium Ecosystem Assessment. Proceedings of the National Academy of Sciences 106(5):1305-1312

Chambers RG (1988) Applied production analysis: A dual approach. Cambridge University Press

Cialdini RB, Goldstein NJ (2004) Social influence: compliance and conformity. Annual Review of Psychology 55:591-621

Contamin R, Ellison AM (2009) Indicators of regime shifts in ecological systems: What do we need to know and when do we need to know it. Ecological Applications 19:799816

Crépin AS, Lindahl T (2009) Grazing games: Sharing common property resources with complex dynamics. Environmental and Resource Economics 44:29-46

Crépin AS, Biggs R, Polasky S, Troell M, de Zeeuw A (2012) Regime shifts and management. Ecological Economics $84: 15-22$

Dakos V, Carpenter S, Cline T, Lahti L (2012a) Early warning signals toolbox. Version 1.0.2, http://earlywarnings.rforge.r-project.org

Dakos V, Carpenter SR, Brock WA, Ellison AM, Guttal V, Ives AR, Kfi S, Livina V, Seekell DA, van Nes EH, Scheffer M (2012b) Methods for detecting early warnings of critical transitions in time series illustrated using simulated ecological data. PLoS ONE 7(7):e41010

Dakos V, van Nes EH, D'Oderico P, Scheffer M (2012c) Robustness of variance and autocorrelation as indicators of critical slowing down. Ecology 93:264-271

Ditlevsen PD, Johnsen SJ (2010) Tipping points: Early warning and wishful thinking. Geophysical Research Letters 37(19):L19703

Ermentrout B (2011) XPPAUT. Version 6.11, http://www.math.pitt.edu/ bard/xpp/xpp.html

Fehr E, Fischbacher U (2002) Why social preferences matter - the impact of non-selfish motives on competition, cooperation, and incentives. The Economic Journal 112(478):C1-C33 
Folke C, Carpenter SR, Walker B, Scheffer M, Chapin T, Rockström J (2010) Resilience thinking: Integrating resilience, adaptability and transformability. Ecology and Society 15:20

Gehrmann E, Drossel B (2010) Boolean versus continuous dynamics on simple two-gene modules. Physical Review E 82:046120

Gross T, Feudel U (2004) Analytical search for bifurcation surfaces in parameter space. Physica D 195(34):292-302

Gross T, Feudel U (2006) Generalized models as a universal approach to the analysis of nonlinear dynamical systems. Physical Review E 73(1):016205

Gross T, Rudolf L, Levin SA, Dieckmann U (2009) Generalized models reveal stabilizing factors in food webs. Science 325:747-750

Guckenheimer J (1978) The catastrophe controversy. The Mathematical Intelligencer 1:15-20

Hastings A, Wysham DB (2010) Regime shifts in ecological systems can occur with no warning. Ecology Letters 13:464-472

Horan RD, Fenichel EP, Drury KLS, Lodge DM (2011) Managing ecological thresholds in coupled environmentalhuman systems. Proc Natl Acad Sci USA 108:7333-7338

Iwasa Y, Uchida T, Yokomizo H (2007) Nonlinear behavior of the socio-economic dynamics for lake eutrophication control. Ecological Economics 63(1):219-229

Kelley WG, Peterson AC (2010) The Theory of Differential Equations: Classical and Qualitative, 2nd edn. Springer

Kline RB (2011) Principles and Practice of Structural Equation Modeling, 3rd edn. Guilford Press, New York

Kuehn C (2011) A mathematical framework for critical transitions: Bifurcations, fast-slow systems and stochastic dynamics. Physica D 240:1020-1035

Kuehn C (2013) A mathematical framework for critical transitions: normal forms, variance and applications, journal of Nonlinear Science, accepted, to appear

Kuehn C, Siegmund S, Gross T (2013) Dynamical analysis of evolution equations in generalized models, IMA Journal of Applied Mathematics, accepted, to appear

Kuznetsov Y (2010) Elements of Applied Bifurcation Theory. Springer

Lade SJ, Gross T (2012) Early warning signals for critical transitions: A generalized modeling approach. PLoS Computational Biology 8(2):e1002360

Lenton TM (2012) What early warning systems are there for environmental shocks? Environmental Science \& Policy In press

Levin S, Xepapadeas T, Crépin AS, Norberg J, de Zeeuw A, Folke C, Hughes T, Arrow K, Barrett S, Daily G, Ehrlich P, Kautsky N, Mäler KG, Polasky S, Troell M, Vincent JR, Walker B (2012) Social-ecological systems as complex adaptive systems: modeling and policy implications. Environment and Development Economics FirstView:1-22

Millennium Ecosystem Assessment (2005) Ecosystems and Human Well-Being: Synthesis. Island Press, Washington DC

Osés-Eraso N, Viladrich-Grau M (2007) On the sustainability of common property resources. Journal of Environmental Economics and Management 53(3):393-410

Ostrom E (1990) Governing the Commons: The Evolution of Institutions for Collective Action. Cambridge University Press

Ostrom E (2006) The value-added of laboratory experiments for the study of institutions and common-pool resources. Journal of Economic Behavior \& Organization 61(2):149163
Perretti CT, Munch SB (2012) Regime shift indicators fail under noise levels commonly observed in ecological systems. Ecological Applications 22:1771-1779

Polasky S, de Zeeuw A, Wagener F (2011) Optimal management with potential regime shifts. Journal of Environmental Economics and Management 62(2):229-240

Raffensperger C, Tickner J (eds) (1999) Protecting Public Health and the Environment: Implementing the Precautionary Principle. Island Press

Scheffer M, Carpenter S, Foley JA, Folke C, Walker B (2001) Catastrophic shifts in ecosystems. Nature 413:591-596

Scheffer M, Bascompte J, Brock WA, Brovkin V, Carpenter SR, Dakos V, Held H, van Nes EH, Rietkerk M, Sugihara G (2009) Early-warning signals for critical transitions. Nature 461:53-59

Scheffer M, Carpenter SR, Lenton TM, Bascompte J, Brock W, Dakos V, van de Koppel J, van de Leemput IA, Levin SA, van Nes EH, Pascual M, Vandermeer J (2012) Anticipating critical transitions. Science 338:344-348

Schlüter M, McAllister RRJ, Arlinghaus R, Bunnefeld N, Eisenack K, Hölker F, Milner-Gulland E, Müller B, Nicholson E, Quaas M, Stöven M (2012a) New horizons for managing the environment: A review of coupled socialecological systems modeling. Natural Resource Modeling $25: 219-272$

Schlüter M, Müller B, Frank K (2012b) MORE - MOdeling for Resilience thinking and Ecosystem stewardship. Available at SSRN: http://ssrn.com/abstract $=2037723$ or http://dx.doi.org/10.2139/ssrn.2037723

Sterman JD (2000) Business Dynamics: Systems Thinking and Modeling for a Complex World. McGraw-Hill

Steuer R, Gross T, Selbig J, Blasius B (2006) Structural kinetic modeling of metabolic networks. Proc Natl Acad Sci 103(32):11868-11873

Tarui N, Mason CF, Polasky S, Ellis G (2008) Cooperation in the commons with unobservable actions. Journal of Environmental Economics and Management 55(1):37-51

Tavoni A, Schlüter M, Levin S (2012) The survival of the conformist: Social pressure and renewable resource management. Journal of Theoretical Biology 299:152-161

Traulsen A, Claussen JC, Hauert C (2005) Coevolutionary dynamics: From finite to infinite populations. Physical Review Letters 95:238701

Zeeman EC (1977) Catastrophe theory: Selected papers, 1972-1977. Addison-Wesley

Zumsande M, Gross T (2010) Bifurcations and chaos in the mapk signaling cascade. Journal of Theoretical Biology 265(3):481-491

Zumsande M, Stiefs D, Siegmund S, Gross T (2011) General analysis of mathematical models for bone remodeling. Bone 48(4):910-917 